\title{
Silvicultural, hydro-orological and economic aspects of a combination of vegetative (Falcataria moluccana-Vigna cylindrica) and terrace systems in soils of different slopes
}

\author{
KARYATI ${ }^{1, \nu}$, SRI SARMINAH $^{1}$, KARMINI $^{2, v \vee}$, RUJEHAN $^{1}$, VEBI FITRIANA EKO LESTARI ${ }^{1}$, \\ WENDY SATRIA PANORAMA ${ }^{1}$ \\ ${ }^{1}$ Faculty of Forestry, Universitas Mulawarman. Jl. Ki Hajar Dewantara, Gunung Kelua, Samarinda 75123, East Kalimantan, Indonesia \\ Tel.: +62-541-35089, Fax.: +62-541-732146, `email: karyati@fahutan.unmul.ac.id, karyati.hanapi@yahoo.com \\ ${ }^{2}$ Faculty of Agriculture, Faculty of Agriculture, UniversitasMulawarman. J1. Pasir Balengkong No.1 Kampus Gunung Kelua, Samarinda75119, East \\ Kalimantan,Indonesia.Tel./Fax.:+62-541-749159/738341, ”vemail: karmini@ faperta.unmul.ac.id, karmini.kasiman@yahoo.com
}

Manuscript received: 12 July 2019. Revision accepted: 25 July 2019.

\begin{abstract}
Karyati, Sarminah S, Karmini, Rujehan, Lestari VFE, Panorama WS. 2019. Silvicultural, hydro-orological and economic aspects of a combination of vegetative (Falcataria moluccana-Vigna cylindrica) and terrace systems in soils of different slopes. Biodiversitas 20: xxxx. Soil and water conservation techniques involving a combination of vegetative and mechanical systems will increase the benefits from both conservation aspect as well as economic aspect. This study was aimed at analyzing the silvicultural, hydro-orological and economic aspects of a combination of vegetative (Falcataria moluccana-Vigna cylindrica) and terrace system in soils of different slopes (a steep and a very steep slope gradient). The silvicultural parameters examined in this study were the ground coverage of $V$. cylindrica growth and survival rate, stem diameter and height of $F$. moluccana trees. The hydro-orological parameters included erosion rate, erosion hazard index, erosion hazard class, and erosion hazard level. The economic parameters included total cost, total revenue, and profit. The result showed that the survival rate, diameter increment and height increment of $F$. moluccana, and ground coverage of $V$. cylindrica in the land with the steep slope (>25-45\%) was $90 \%, 2.02 \mathrm{~cm}_{\text {year }}{ }^{-1}, 1.54 \mathrm{~m}_{\text {year }}{ }^{-1}$, and $80-90 \%$, respectively. The erosion rate, erosion hazard index, erosion hazard class, and erosion hazard level in this steep slope was 0.38 ton ha year-1 $^{-1} 0.03$ (low), I (very low), and very low, respectively. In the steeper ground (>45\%), the survival rate of $F$. moluccana reached $90 \%$, the $V$. cylindrica coverage was 70-79\% and the diameter and height increment of $F$. moluccana was $1.63 \mathrm{~cm}^{\text {year }}{ }^{-1}$ and $1.19 \mathrm{~m} \mathrm{year}^{-1}$,

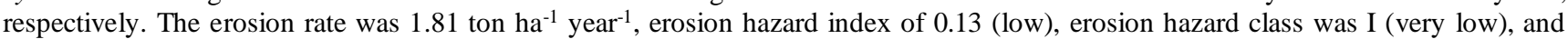
erosion hazard level was low in the very steep slope land. The profit from $V$. cylindrica was Rp 3,865,000.00 ha ${ }^{-1} \mathrm{cropping} \mathrm{season}^{-1}$ and $\mathrm{Rp} 665,000.00 \mathrm{ha}^{-1}$ cropping season $^{-1}$ in steep slope and very steep slope, respectively. The application of the proposed combination of vegetative and terrace system could reduce surface runoff and erosion rate in the long term, in addition to providing short term economic benefits.
\end{abstract}

Keywords: Economic, hydro-orological, silvicultural, slope, terrace

\section{INTRODUCTION}

The overexploitation of natural resources which has exceeded the carrying capacity, coupled with population pressure on land resources, will lead to an increase in degraded land area. In Indonesia, 78 million ha area of land has been categorized as degraded land, of which, 48 million ha is slightly degraded area, 23 million ha is moderately degraded area and 7 million ha is highly degraded area (ADB 2016). The implementation of soil and water conservation and other land rehabilitation at in situ and catchment level is the highest priority to manage highly degraded environments and pressured lands (Nyssen et al. 2008). The application of vegetative barriers and mechanical technique, such as bunds or trenches or both, on the appropriately spaced contours, may increase potential conservation role (Dass et al. 2011).

Soil reaction primarily influences plant growth indirectly through its effects on the solubility of ions and the activity of microorganisms (Harris 1992). The easily observable properties of soil, such as texture, structure, color, depth, and stoniness, can be used to infer a great deal about how a particular soil influences plant growth (Fisher and Binkley 2000). It is the ability of the soil to supply nutrient elements in the amounts, forms, and proportions required for maximum plant growth (Hazra and Som 2006). There is association between species and soil characteristics under homogenous parent rock and elevational range (Nizam et al. 2006) as well as correlation among topography, soil nutrient and plants (Potts et al. 2002). In addition, several soil properties showed positive and negative correlation to plant parameters (Kumar et al. 2010; Toledo et al. 2011).

The mixed cropping of sengon (Falcataria moluccana) and peanut (Arachis hypogaea) as well as jabon (Anthocephalus cadamba) and soybeans (Glycine max) could be implemented in different soil slopes for rehabilitating and soil conservation in sloping lands, based on the growth and hydro-orological parameters (Karyati et al. 2018; Sarminah et al. 2018). These two agroforestry systems of F. moluccana-A. hypogaea and A. cadamba-G. max are feasible and applicable to rehabilitate and conserve 
the critical lands. Although planting of $G$. max was not profitable in some critical lands, the agroforestry system of A.cadamba and G. max still had many benefits from the aspect of ecology and conservation for long terms (Karmini et al. 2017).

Yard long bean (Vigna sesquipedalis L. Fruw) is one of the most popular vegetables in many countries of Southeast Asia, but this species has relatively low yield productivity (Nooprom and Santipracha 2015). The production, harvested area, and productivity of Vigna cylindrica (long beans) in Indonesia in 2017 was 381,185 ton; 56,111 hectare, and 6.79 ton hectare ${ }^{-1}$, respectively (Ministry of Agriculture Republic of Indonesia 2018). In East Kalimantan, the production of $V$. cylindrica in 2017 was 71,456 quintal, while harvested area and productivity was 1,361 hectare and 52.50 quintal hectare ${ }^{-1}$. The productivity of $V$. cylindrica in Samarinda in 2017 was 26.86 quintal hectare $^{-1}$, total production was 3,089 quintal and the harvested area of 115 hectares (Ministry of Agriculture Republic of Indonesia 2018; Statistics of East Kalimantan Province 2018).

The total land area of Samarinda City of East Kalimantan Province is $71.800,00$ ha, out of which land area with less than $2 \%$ slope is $27.39 \%, 2-15 \%$ slope is $25.47 \%, 15-25 \%$ slope is $14.81 \%, 25-40 \%$ slope is $15.67 \%$, and more than $40 \%$ slope is $13.02 \%$ (Statistics of East Kalimantan Province 2018). A combination of vegetative and terrace techniques of soil and water conservation may be the appropriate choice to rehabilitate sloping lands based on silvicultural, hydro-orological and economic aspects. The plantations of forestry plants is hoped to provide silvicultural, conservation and economic values in a long term program. On the other hand, planting of agricultural plants is expected to provide short term economic benefits. The objectives of this study were to investigate silvicultural, hydro-orological and economic aspects of implementation of a combination of vegetative ( $F$. moluccana- $V$. cylindrica) and terrace systems in different soil slopes, ranging from steep to very steep slope gradients.

\section{MATERIALS AND METHODS}

\section{Study area}

This study was conducted in Educational Forest of Forestry Faculty of Mulawarman University, Lempake, Samarinda City, East Kalimantan Province, Indonesia. The study duration was six months, from January to June 2018. The experimental forest covered a total area of 300 ha and was located at $0^{\circ} 25^{\prime} 10^{\prime \prime}-0^{\circ} 25^{\prime} 24^{\prime \prime}$ South latitude and $117^{\circ} 14^{\prime} 00^{\prime \prime}-117^{\circ} 14^{\prime} 14^{\prime \prime}$ East longitude, in between kilometers 10 and 13 of the Samarinda-Bontang Highway. Administratively, this experimental forest is situated in Tanah Merah Village, Samarinda City, East Kalimantan Province, Indonesia.

The study area received $211.5 \mathrm{~mm}$ monthly average rainfall, average air temperature is $27.4^{\circ} \mathrm{C}$, and relative humidity was $82.2 \%$. The average daily temperature and relative humidity inside the forest ranged from $23.7-30.9^{\circ} \mathrm{C}$ and 81.4-99.3\%, respectively. The average daily temperature and relative humidity outside the forest was $25.9^{\circ} \mathrm{C}-28.8^{\circ} \mathrm{C}$ and $76.0 \%-90.0 \%$, respectively. The daily average light intensity ranged from $1.08 \mu \mathrm{mol}$ to 18.41 $\mu \mathrm{mol}$ (Karyati and Ardianto 2016). The climate of Samarinda City was classified as type A climate (a quotient (Q) of $4.8 \%$ ) based on Schmidt and Ferguson (1951) system which is characterized by high humidity with a tropical rainforest vegetation.

\section{Procedures \\ Experimental procedures}

Experiments were conducted in two $10 \mathrm{~m} \mathrm{x} 10 \mathrm{~m}$ experimental plots which were located in areas with two different slope classes inside the experimental forest. One of these two experimental plots had a steep slope $(>25$ $45 \%)$ and others had a very steep slope $(>45 \%)$.

Two-meters $(2 \mathrm{~m})$ wide terraces were made in each experimental plot. Terrace was completed by ditches sized $25 \mathrm{~cm}$ wide and $10 \mathrm{~cm}$ depth. Sengon ( $F$. moluccana) and long beans ( $V$. cylindrica) were planted on both plots. $F$. moluccana trees were planted with a spacing of $3 \mathrm{~m} \times 3 \mathrm{~m}$. $V$. cylindrica, as the intercrop legume, was grown in between the sengon trees. Two erosion measurement plots of $10 \mathrm{~m} \times 3 \mathrm{~m}$ size were placed in each of the two experimental plots. The growth parameters were measured once in every month, for four months period. Plants were maintained by regular watering, weeding, fertilizer application, and pest and disease control. For economic evaluation, only the long beans were harvested, but not the sengon trees. The measurement of hydro-orological parameters was conducted during 27 times rain events.

\section{Analysis of soil properties}

The analysis of soil physicochemical properties, such as $\mathrm{pH}, \mathrm{C}$ organic, total $\mathrm{N}, \mathrm{P}, \mathrm{K}$, and soil texture, were conducted in Laboratory of Soil Science, Tropical Forest Research Center, Mulawarman University. Soil pH was determined in distilled water and $1 \mathrm{~N} \mathrm{KCl}$ in soil to solution ratio of $1: 2.5$ by the glass electrode method. The $\mathrm{C}$ organic and total nitrogen (total $\mathrm{N}$ ) were analyzed using Walkley and Black method (1934) and Kjeldahl method (1883), respectively. Soil P and K were analyzed using Bray 1 method (1954).

\section{Data analysis \\ Silvicultural parameters}

The observation and measurement of plant growth were conducted at the end of every month for four months. The observation was carried out on both $F$. moluccana and $V$. cylindrica plants. The parameters studied for $F$. moluccana were plant survival rate, tree height, and stem diameter. For $V$. cylindrica, ground coverage and yield was recorded. The plant health was measured for both $F$. moluccana and $V$. cylindrica. A healthy plant was characterized as a plant with a normal height, fresh green leaves, normal stem, and no disease/pests and weed (Ministry of Forestry Republic of Indonesia 2009). 


\section{Hydro-orological parameter}

The hydro-orological parameters measured in this study were surface runoff, potential soil erosion rate, erosion hazard index, erosion hazard class, and erosion hazard level (Hammer 1981). The erosion hazard index categories and erosion hazard level classification are shown in Table 1 and Table 2, respectively. The erosion hazard index is determined as potential erosion rate (ton $\mathrm{ha}^{-1}$ year $^{-1}$ ) divided by tolerable erosion rate (ton ha $^{-1}$ year $^{-1}$ ) (Hammer 1981).

\section{Economic analysis}

In this study, the analyzed economic aspects were cost, revenue and profit from long beans as intercropping in sengon (long beans) agroforestry systems. Cost is calculated from price and quantity of inputs, revenue is the price of produced yield, and profit is revenue minus cost (Slavin 2009).

\section{RESULTS AND DISCUSSION}

\section{Silvicultural aspects}

The recorded plant growth parameters of $F$. moluccana and $V$. cylindrica for the two different slope classes are summarized in Table 3. The growth of F. moluccana and $V$. cylindrica combination were "moderate" to "very good" in the steep slope and very steep slope. The survival rate of F. moluccana in both slope classes was "very good" (90\%). The ground coverage of $V$. cylindrica in steep slope was better $(80-89 \%)$ than in very steep slope (70-79\%). Meanwhile, the yield of $V$. cylindrica was also higher $\left(2,500 \mathrm{~kg} \mathrm{ha}^{-1}\right)$ in steep slope when compared to the yield in very steep slope $\left(2,100 \mathrm{~kg} \mathrm{ha}^{-1}\right)$.

The average of $V$. cylindrica yield in this study was almost similar to the average of Samarinda's yield, but lower than East Kalimantan and Indonesia's yield (Ministry of Agriculture Republic of Indonesia 2018; Statistics of East Kalimantan Province 2018). However, $V$. cylindrica's yield obtained in this study was lower than reported by Wahyu et al. (2018). Wahyu et al. (2018) reported that the productivity of $V$. cylindrica in Samboja Subdistrict, Kutai Kartanegara District, East Kalimantan Province was 7,010 $\mathrm{kg} \mathrm{ha}^{-1}$ and $13,640 \mathrm{~kg} \mathrm{ha}^{-1}$ from monoculture and agroforestry systems, respectively.

The monthly diameter and height increments of $F$. moluccana trees, measured for four months, are provided in
Tables 4 and 5. The stem diameter and height increment of $F$. moluccana trees is faster in the slightly steep slope than in the highly steeper slope. The average stem diameter and height increments of $F$. moluccana were $2.02 \mathrm{~cm} \mathrm{year}^{-1}$ and $1.54 \mathrm{~m} \mathrm{year}^{-1}$ in the steep slope, and $1.63 \mathrm{~cm} \mathrm{year}^{-1}$ and $1.19 \mathrm{~m} \mathrm{year}^{-1}$, in very steep slope.

The average diameter increment of $F$. moluccana in $F$. moluccana and $V$. cylindrica agroforestry system (on steep and very steep slopes) was lower than those in $F$. moluccana and Arachis hypogaea agroforestry system (on slight steep and steep slopes) as reported by Sarminah et al. (2018). On the contrary, the average height increment of this combination was higher than those reported by Sarminah et al. (2018). Similarly, the diameter increment of $F$. moluccana in this study was also lower than those in agroforestry system, monoculture system, and intensive monoculture system (Swestiani and Purwaningsih 2013; Wahyudi and Panjaitan 2013). However, the average diameter increments of $F$. moluccana trees on the steep slope was higher than those planted in the conventional monoculture system (Wahyudi and Panjaitan 2013).

\section{Hydro-orological aspects}

The hydro-orological aspects were determined by surface runoff and eroded soil mass. Table 6 presents data regarding rainfall, eroded soil mass, and surface runoff volume in vegetative ( $F$. moluccana-V.cylindrica) and terrace combination system in the two different slope classes. The result showed that the higher rainfall results in surface runoff and eroded soil mass. Several factors such as rainfall, slope, cover crop, and management practices have suspected influence on soil erosion in the study sites. The length and gradient of slope influence soil erosion. In the steeper slope lands, the rainfall tends to transport the soil particles into lower area faster. It will increase surface runoff and eroded soil mass as well as erosion rate. The increasing slope and rain intensity have increased the runoff rate from $20 \%$ to $90 \%$ (Chaplot and LeBissonnais 2000)

Table 1. Erosion hazard index categories

\begin{tabular}{cc}
\hline Erosion hazard index & Category \\
\hline$<1.00$ & Low \\
$1.01-4.00$ & Moderate \\
$4.01-10.00$ & High \\
$>10.01$ & Very high \\
\hline
\end{tabular}

Source: Hammer (1981)

Table 2. Erosion hazard level classification

\begin{tabular}{|c|c|c|c|c|c|}
\hline \multirow{4}{*}{ Soil column $(\mathbf{c m})$} & \multicolumn{5}{|c|}{ Erosion class } \\
\hline & I & II & III & IV & $\mathbf{V}$ \\
\hline & \multicolumn{5}{|c|}{ Erosion rate (ton ha ${ }^{-1}$ year $\left.^{-1}\right)$} \\
\hline & $<15$ & $15-<60$ & $60-<180$ & $180-480$ & $>480$ \\
\hline Deep $(>90)$ & Very low 0 & Low I & Moderate II & Heavy III & Very heavy IV \\
\hline Intermediate $(60-90)$ & Low I & Moderate II & Heavy III & Very high IV & Very heavy IV \\
\hline Shallow $(30-<60)$ & Moderate II & Heavy III & Very heavy IV & Very heavy IV & Very heavy IV \\
\hline Very shallow $(<30)$ & Heavy III & Very heavy IV & Very heavy IV & Very heavy IV & Very heavy IV \\
\hline
\end{tabular}

Source: Regulation of Directorate General of Watershed Management and Social Forestry, Ministry of Forestry Republic of Indonesia (2013). Note: 0: Very low; I: Low; II: Moderate; III: Heavy; IV: Very heavy 
Table 3. The growth parameters of F. moluccana and $V$. cylindrica in the two different slope classes

\begin{tabular}{|c|c|c|c|c|c|c|c|c|}
\hline \multirow[b]{2}{*}{ Plant species } & \multicolumn{4}{|c|}{ Steep slope (>25-45\%) } & \multicolumn{4}{|c|}{ Very steep slope (>45\%) } \\
\hline & $\begin{array}{l}\text { Healthy } \\
\text { plants }(\%)\end{array}$ & $\begin{array}{l}\text { Survival } \\
\text { rate }(\%)\end{array}$ & $\begin{array}{c}\text { Ground } \\
\text { coverage }(\%)\end{array}$ & $\begin{array}{c}\text { Yield } \\
\left(\mathrm{kg} \mathrm{ha}^{-1}\right)\end{array}$ & $\begin{array}{c}\text { Healthy } \\
\text { plants }(\%)\end{array}$ & $\begin{array}{l}\text { Survival } \\
\text { rate }(\%)\end{array}$ & $\begin{array}{c}\text { Ground } \\
\text { coverage }(\%)\end{array}$ & $\begin{array}{c}\text { Yield } \\
\left(\mathrm{kg} \mathrm{ha}^{-1}\right)\end{array}$ \\
\hline F. moluccana & $\begin{array}{c}90 \\
\text { (Very good) }\end{array}$ & $\begin{array}{c}90 \\
\text { (Very good) }\end{array}$ & - & - & $\begin{array}{c}90 \\
\text { (Very good) }\end{array}$ & $\begin{array}{c}90 \\
\text { (Very good) }\end{array}$ & - & - \\
\hline V. cylindrica & $80-89$ (Good) & - & $\begin{array}{l}80-89 \\
\text { (Good) }\end{array}$ & 2,500 & $\begin{array}{c}70-79 \\
\text { (Moderate) }\end{array}$ & - & $\begin{array}{c}70-79 \\
\text { (Moderate) }\end{array}$ & 2,100 \\
\hline
\end{tabular}

Table 4. Falcataria moluccana stem diameter increments $(\mathrm{mm})$ in the two different slopes classes

\begin{tabular}{|c|c|c|c|c|c|c|c|c|c|c|}
\hline \multirow{2}{*}{$\begin{array}{c}\text { Tree } \\
\text { number }\end{array}$} & \multicolumn{5}{|c|}{ Steep slope (>25-45\%) } & \multicolumn{5}{|c|}{ Very steep slope (>45\%) } \\
\hline & Do & $d_{1}$ & $d_{2}$ & d3 & $d_{4}$ & Do $_{0}$ & $d_{1}$ & $\mathbf{d}_{2}$ & d3 & $d_{4}$ \\
\hline 1 & 12.10 & 12.85 & 14.15 & 16.15 & 18.68 & 14.40 & 15.00 & 16.05 & 18.25 & 21.10 \\
\hline 2 & 9.25 & 10.00 & 11.25 & 12.80 & 15.00 & 6.20 & 6.95 & 7.90 & 9.25 & 11.00 \\
\hline 3 & 8.20 & 8.90 & 10.10 & 11.65 & 13.85 & 9.10 & 9.70 & 11.00 & 12.95 & 15.50 \\
\hline 4 & 5.10 & 7.80 & 9.05 & 10.60 & 12.50 & 9.70 & 10.20 & 11.25 & 12.75 & 15.10 \\
\hline 5 & 12.70 & 13.65 & 14.95 & 16.90 & 19.25 & 11.10 & 11.65 & 12.90 & 14.85 & 17.35 \\
\hline 6 & 8.60 & 9.55 & 11.00 & 12.70 & 14.75 & 6.75 & 7.35 & 8.30 & 9.60 & 11.25 \\
\hline 7 & 5.30 & 8.00 & 9.20 & 10.90 & 13.00 & 6.60 & 7.30 & 8.40 & 9.85 & 11.70 \\
\hline 8 & 7.40 & 8.15 & 9.40 & 11.05 & 13.00 & 6.40 & 7.00 & 7.95 & 9.40 & 11.15 \\
\hline 9 & 10.30 & 10.90 & 12.05 & 13.55 & 15.50 & 8.40 & 9.00 & 9.95 & 11.30 & 13.00 \\
\hline 10 & 10.10 & 10.80 & 12.20 & 13.90 & 16.10 & 10.40 & 11.10 & 12.15 & 14.05 & 16.45 \\
\hline 11 & 5.70 & 8.50 & 9.75 & 11.40 & 13.55 & 8.20 & 8.85 & 9.80 & 11.15 & 12.90 \\
\hline 12 & 10.30 & 11.20 & 12.45 & 14.20 & 16.60 & 9.10 & 10.00 & 11.50 & 13.30 & 15.65 \\
\hline 13 & 11.40 & 12.10 & 13.40 & 15.55 & 18.20 & 7.10 & 7.75 & 8.80 & 10.30 & 12.05 \\
\hline 14 & 6.30 & 9.10 & 10.20 & 12.05 & 14.15 & 10.35 & 11.00 & 12.20 & 13.70 & 15.65 \\
\hline 15 & 10.80 & 11.50 & 12.90 & 14.95 & 17.55 & 7.25 & 8.00 & 9.10 & 10.50 & 12.20 \\
\hline 16 & 4.15 & 8.75 & 9.75 & 11.55 & 13.65 & 14.40 & 15.15 & 16.50 & 18.29 & 20.19 \\
\hline Mean & 8.61 & 10.11 & 11.36 & 13.12 & 15.33 & 9.09 & 9.75 & 10.86 & 12.47 & 14.52 \\
\hline SD & 2.69 & 1.81 & 1.86 & 1.99 & 2.17 & 2.58 & 2.58 & 2.66 & 2.88 & 3.16 \\
\hline \multicolumn{2}{|c|}{$\begin{array}{l}\text { Annual diameter } \\
\text { increment }\end{array}$} & \multicolumn{4}{|c|}{$20.18 \mathrm{~mm}_{\text {year }}{ }^{-1}=2.02 \mathrm{~cm}_{\text {year }}{ }^{-1}$} & $\begin{array}{l}\text { Annual dian } \\
\text { increment }\end{array}$ & \multicolumn{4}{|c|}{$16.27 \mathrm{~mm} \mathrm{year}^{-1}=1.63 \mathrm{~cm}$ year $^{-1}$} \\
\hline
\end{tabular}

Table 5. Falcataria moluccana height increments $(\mathrm{cm})$ in the two different slopes

\begin{tabular}{|c|c|c|c|c|c|c|c|c|c|c|}
\hline \multirow{2}{*}{$\begin{array}{c}\text { Tree } \\
\text { number }\end{array}$} & \multicolumn{5}{|c|}{ Steep slope (>25-45\%) } & \multicolumn{5}{|c|}{ Very steep slope (>45\%) } \\
\hline & $\mathbf{H}_{0}$ & h1 & $\mathbf{h}_{2}$ & h3 & h4 & $\mathbf{H}_{0}$ & h1 & $\mathbf{h}_{2}$ & h3 & h4 \\
\hline 1 & 153 & 177 & 189 & 205 & 213 & 176 & 197 & 205 & 210 & 220 \\
\hline 2 & 144 & 157 & 182 & 194 & 206 & 160 & 173 & 182 & 201 & 205 \\
\hline 3 & 102 & 106 & 111 & 117 & 125 & 143 & 153 & 157 & 164 & 174 \\
\hline 4 & 176 & 188 & 196 & 202 & 212 & 124 & 136 & 148 & 155 & 166 \\
\hline 5 & 165 & 183 & 204 & 212 & 227 & 146 & 163 & 166 & 171 & 176 \\
\hline 6 & 157 & 172 & 194 & 204 & 212 & 123 & 138 & 145 & 158 & 161 \\
\hline 7 & 167 & 179 & 187 & 200 & 209 & 145 & 157 & 167 & 176 & 188 \\
\hline 8 & 146 & 167 & 183 & 192 & 204 & 151 & 165 & 178 & 182 & 194 \\
\hline 9 & 182 & 205 & 219 & 231 & 242 & 146 & 162 & 166 & 175 & 181 \\
\hline 10 & 184 & 197 & 208 & 219 & 231 & 164 & 177 & 186 & 199 & 206 \\
\hline 11 & 116 & 131 & 143 & 159 & 177 & 173 & 185 & 189 & 194 & 208 \\
\hline 12 & 119 & 136 & 148 & 163 & 176 & 152 & 163 & 169 & 175 & 184 \\
\hline 13 & 159 & 173 & 183 & 195 & 208 & 119 & 134 & 142 & 154 & 162 \\
\hline 14 & 168 & 184 & 194 & 212 & 220 & 169 & 186 & 190 & 202 & 214 \\
\hline 15 & 169 & 185 & 198 & 211 & 221 & 134 & 149 & 158 & 167 & 178 \\
\hline 16 & 137 & 150 & 163 & 172 & 181 & 101 & 113 & 126 & 136 & 144 \\
\hline Mean & 153 & 168 & 181 & 193 & 204 & 145 & 159 & 167 & 176 & 185 \\
\hline SD & 24.10 & 26.21 & 27.59 & 28.10 & 28.03 & 21.03 & 22.03 & 20.79 & 20.69 & 21.48 \\
\hline \multicolumn{2}{|l|}{$\begin{array}{l}\text { Annual height } \\
\text { increment }\end{array}$} & \multicolumn{4}{|c|}{$153.75 \mathrm{~cm}$ year $^{-1}=1.54 \mathrm{~m}$ year $^{-1}$} & $\begin{array}{l}\text { Annual } 1 \\
\text { incremer }\end{array}$ & & \multicolumn{3}{|c|}{$119.1 \mathrm{~cm} \mathrm{year}^{-1}=1.19 \mathrm{~m}$ year $^{-1}$} \\
\hline
\end{tabular}

Note: $\mathrm{H}_{0}$ : initial tree height (height measurement at the beginning of experiment); $\mathrm{h}_{1}, \mathrm{~h}_{2}, \mathrm{~h}_{3}, \mathrm{~h}_{4}$ : height at the end of the first, second, third, and fourth month after planting; SD: Standard Deviation 
Table 6. Rainfall, surface runoff volume, and eroded soil mass in vegetative (F. moluccana-V.cylindrica) and terrace combination system in the two different slope classes

\begin{tabular}{|c|c|c|c|c|c|}
\hline \multirow[b]{2}{*}{ Rain event } & \multirow{2}{*}{$\begin{array}{c}\text { Rainfall } \\
\text { (mm) }\end{array}$} & \multicolumn{2}{|c|}{ Surface runoff $(\mathrm{L})$} & \multicolumn{2}{|c|}{ Eroded soil mass $\left(\mathrm{g} / 30 \mathrm{~m}^{2}\right)$} \\
\hline & & $\begin{array}{l}\text { Steep slope } \\
(>25-45 \%)\end{array}$ & $\begin{array}{c}\text { Very steep slope } \\
(>45 \%)\end{array}$ & $\begin{array}{r}\text { Steep slope } \\
(>25-45 \%)\end{array}$ & $\begin{array}{c}\text { Very steep slope } \\
(>45 \%)\end{array}$ \\
\hline 1 & 74 & 46.74 & 43.40 & 128.76 & 111.59 \\
\hline 2 & 37 & 12.21 & 46.74 & 39.93 & 631.4 \\
\hline 3 & 14 & 7.87 & 46.74 & 13.51 & 149.74 \\
\hline 4 & 20 & 7.87 & 46.74 & 9.22 & 164.05 \\
\hline 5 & 41 & 46.74 & 46.26 & 119.22 & 348.13 \\
\hline 6 & 21 & 14.95 & 47.21 & 47.72 & 214.6 \\
\hline 7 & 36 & 46.74 & 46.74 & 62.95 & 147.84 \\
\hline 8 & 22 & 15.74 & 46.74 & 32.75 & 115.41 \\
\hline 9 & 7 & 4.72 & 7.79 & 4.96 & 25.43 \\
\hline 10 & 15 & 6.30 & 46.74 & 11.06 & 97.29 \\
\hline 11 & 13 & 6.23 & 15.74 & 6.49 & 105.87 \\
\hline 12 & 22 & 23.37 & 47.21 & 14.31 & 75.35 \\
\hline 13 & 31 & 46.74 & 47.21 & 32.43 & 61.04 \\
\hline 14 & 49 & 11.8 & 46.74 & 18.12 & 96.33 \\
\hline 15 & 10 & 6.3 & 47.21 & 2.8 & 57.23 \\
\hline 16 & 10 & 6.3 & 17.92 & 2.03 & 15.36 \\
\hline 17 & 17 & 7.87 & 46.74 & 2.07 & 22.89 \\
\hline 18 & 5 & 8.66 & 30.69 & 0.7 & 24.18 \\
\hline 19 & 9 & 4.72 & 31.48 & 0.38 & 8.9 \\
\hline 20 & 14 & 7.87 & 46.74 & 0.16 & 15.26 \\
\hline 21 & 28 & 7.87 & 46.74 & 2.07 & 34.34 \\
\hline 22 & 41 & 14.16 & 47.21 & 1.14 & 14.31 \\
\hline 23 & 42 & 37.77 & 47.21 & 3.05 & 35.29 \\
\hline 24 & 21 & 11.02 & 47.21 & 1.11 & 25.75 \\
\hline 25 & 27 & 11.80 & 46.74 & 0.72 & 16.21 \\
\hline 26 & 10 & 7.87 & 47.21 & 0.16 & 11.45 \\
\hline 27 & 45 & 15.74 & 47.21 & 0.32 & 8.58 \\
\hline Total & 681 & 445.92 & 1132.26 & 558.13 & 2633.81 \\
\hline Average & 25 & 16.52 & 41.94 & 20.67 & 97.55 \\
\hline
\end{tabular}

Table 7. The hydro-orological parameters in vegetative (F. moluccana-V.cylindrica) and terrace combination system in the two different slope classes

\begin{tabular}{|c|c|c|c|c|c|c|}
\hline $\begin{array}{l}\text { Slope } \\
\text { gradient }\end{array}$ & $\begin{array}{l}\text { Surface runoff rate } \\
\left(\mathbf{m}^{3} \mathbf{h a}^{-1} \text { year }^{-1}\right)\end{array}$ & $\begin{array}{c}\text { Potential erosion } \\
\text { rate } \\
\left(\text { ton } \text { ha }^{-1} \text { year }^{-1}\right)\end{array}$ & $\begin{array}{c}\text { Tolerable } \\
\text { erosion rate } \\
\left(\text { ton }^{-1} \text { year }^{-1}\right)\end{array}$ & $\begin{array}{l}\text { Erosion hazard } \\
\text { index }\end{array}$ & $\begin{array}{c}\text { Erosion } \\
\text { hazard } \\
\text { class }\end{array}$ & $\begin{array}{c}\text { Erosion } \\
\text { hazard } \\
\text { level }\end{array}$ \\
\hline$>25-45 \%$ & 305.77 & 0.38 & $25^{1)}$ & 0.03 (Low) & I (Very low) & Very low \\
\hline$>45 \%$ & 776.41 & 1.81 & $25^{1)}$ & 0.13 (Low) & I (Very low) & Vey low \\
\hline
\end{tabular}

Note: ${ }^{1}$ Soil depth in the study plot was $>100 \mathrm{~cm}$ and the tolerable erosion rate for hills or slope lands was 25 ton ha $^{-1} \mathrm{year}^{-1}(\mathrm{Rahim}$ 1995)

Table 8. Comparison of soil erosion reported in the current study with earlier reports for other agroforestry plantation systems

\begin{tabular}{|c|c|c|c|}
\hline Planting system & $\begin{array}{c}\text { Erosion } \\
\left(\text { ton }^{-1} \text { year }^{-1}\right)\end{array}$ & Location & Researcher (year) \\
\hline $\begin{array}{l}\text { Soil and water conservation technique and application of } \\
\text { agroforestry system }\end{array}$ & 190.08 & $\begin{array}{l}\text { Ngadipiro Village. Nguntoronadi } \\
\text { Sub-district. Wonogiri District. } \\
\text { Central Java. Indonesia }\end{array}$ & Sumarno et al. (2011) \\
\hline Agroforestry system of A. cadamba and Glycine $\max$ & & East Kalimantan. Indonesia & Karyati et al. (2018) \\
\hline Slope of $>15-25 \%$ & 32.13 & & \\
\hline Slope of $>25-45 \%$ & 52.51 & & \\
\hline Agroforestry system of $F$. moluccana and A. hypogaea & & East Kalimantan. Indonesia & Sarminah et al. (2018) \\
\hline Slope of $>15-25 \%$ & 20.05 & & \\
\hline Slope of $>25-45 \%$ & 45.50 & & \\
\hline Agroforestry system of $F$. moluccana and $V$. cylindrica & & East Kalimantan. Indonesia & Current study \\
\hline Slope of $>25-45 \%$ & 0.38 & & \\
\hline Slope of $>45 \%$ & 1.81 & & \\
\hline
\end{tabular}


Table 9. Economic analysis of growing $V$. cylindrica as intercrop in vegetative ( $F$. moluccana- $V$. cylindrica) and terrace combination system in the two different slope classes

\begin{tabular}{|c|c|c|c|c|c|}
\hline No. & Item/activity & Quantity & Unit & Price (Rp) & Total (Rp. ha $\left.{ }^{-1} \mathrm{cs}^{-1}\right)$ \\
\hline \multicolumn{6}{|c|}{ Production cost } \\
\hline \multicolumn{6}{|c|}{ Material cost } \\
\hline 1 & F. moluccana seedlings & $1,350.00$ & units ha-1 & $3,000.00$ & $4,050,000.00$ \\
\hline 2 & V. cylindrica seeds & 350.00 & units ha-1 $\mathrm{cs}^{-1}$ & $15,000.00$ & $5,250,000.00$ \\
\hline 3 & NPK fertilizer & 100.00 & $\mathrm{~kg} \mathrm{ha}^{-1} \mathrm{cs}^{-1}$ & $15,000.00$ & $1,500,000.00$ \\
\hline 4 & Pesticides & 25.00 & $\mathrm{~kg} \mathrm{ha}^{-1} \mathrm{cs}^{-1}$ & $30,000.00$ & $750,000.00$ \\
\hline 5 & Plastic strings & 1.00 & units ha ${ }^{-1} \mathrm{cs}^{-1}$ & $30,000.00$ & $30,000.00$ \\
\hline 6 & Gunny sacks & 20.00 & units ha ${ }^{-1} \mathrm{cs}^{-1}$ & $2,000.00$ & $40,000.00$ \\
\hline \multirow[t]{2}{*}{7} & Fertilizer & 50.00 & units ha ${ }^{-1} \mathrm{cs}^{-1}$ & $25,000.00$ & $1,250,000.00$ \\
\hline & Subtotal & & & & $12,870,000.00$ \\
\hline \multicolumn{6}{|c|}{ Depreciation cost } \\
\hline 8 & Hoe & 2.00 & units ha-1 & $125,000.00$ & $20,833.33$ \\
\hline 9 & Chopper & 2.00 & units ha ${ }^{-1}$ & $100,000.00$ & $16,666.67$ \\
\hline 10 & Sickle & 2.00 & units ha-1 & $60,000.00$ & $10,000.00$ \\
\hline \multirow[t]{2}{*}{11} & Sprayer & 1.00 & units ha-1 & $350,000.00$ & $17,500.00$ \\
\hline & Subtotal & & & & $65,000.00$ \\
\hline \multicolumn{6}{|c|}{ Labor cost } \\
\hline 12 & Land preparation & 7.00 & days $\mathrm{ha}^{-1} \mathrm{cs}^{-1}$ & $100,000.00$ & $700,000.00$ \\
\hline 13 & Planting & 6.00 & days ha-1 $\mathrm{cs}^{-1}$ & $100,000.00$ & $600,000.00$ \\
\hline 14 & Fertilizing & 4.00 & days $\mathrm{ha}^{-1} \mathrm{cs}^{-1}$ & $100,000.00$ & $400,000.00$ \\
\hline 15 & Weeding & 5.00 & days ha-1 $\mathrm{cs}^{-1}$ & $100,000.00$ & $500,000.00$ \\
\hline 16 & Pest and disease controlling & 4.00 & days ha- $\mathrm{cs}^{-1}$ & $100,000.00$ & $400,000.00$ \\
\hline \multirow[t]{2}{*}{17} & Harvesting & 6.00 & days $h^{-1} \mathrm{cs}^{-1}$ & $100,000.00$ & $600,000.00$ \\
\hline & Subtotal & & & & $3,200,000.00$ \\
\hline \multicolumn{5}{|c|}{ Total cost } & $16,135,000.00$ \\
\hline \multicolumn{6}{|c|}{ Total revenue from $V$. cylindrica } \\
\hline & Steep slope plot (>25-45\%) & $2,500.00$ & $\mathrm{~kg} \mathrm{ha}^{-1}$ & $8,000.00$ & $20,000,000.00$ \\
\hline & Very steep slope plot $(>45 \%)$ & $2,100.00$ & $\mathrm{~kg} \mathrm{ha}^{-1}$ & $8,000.00$ & $16,800,000.00$ \\
\hline \multirow[t]{2}{*}{ Profit } & & \multicolumn{2}{|c|}{ Steep slope plot $(>25-45 \%)$} & & $3,865,000.00$ \\
\hline & & Very steep slope & plot $(>45 \%)$ & & $665,000.00$ \\
\hline
\end{tabular}

Note: cs: cropping season

The implementation of vegetative and terrace system was hoped to reduce the potential soil erosion. Generally, the best recommendation for soil and water conservation technology was to combine the vegetative and mechanical systems because such combinations are usually best for reducing soil erosion and decreasing conservation cost in long term as well as increasing short term economic benefits. The $F$. moluccana trees and $V$. cylindrica play a role as cover crop in the sloping lands that could avoid destructive effect of rainfall. In addition, the application of terrace system could reduce surface runoff and increase water infiltration into the deeper soils. Terrace agriculture could be adapted as a land-management practice and innovation in terms of the region and local conditions (Bocco and Napoletano 2017).

The erosion rate is also affected by soil properties, especially soil texture. The soil texture in the study site is silty loam that usually supports the greatest diversity of plant life. The silt tends to be loaded with the soluble nutrients required by plants. The silt soil also has high content of organic matter. The soil in the study plots was categorized as acidic soil, indicated by the $\mathrm{pH}$ value of lower than 5 and the low content of organic C, total N, P, and K. Tanaka (1999) stated the soil acidity could be a good indicator for the composition of exchangeable cations and it provided the capacity of the deeper surface to supply exchangeable bases to plant. It was regardless that the acidic nature of the soils might be due to the loss of exchangeable bases through uptake by plant and leaching under tropical environment (Hamzah et al. 2009)

The evaluation of erosion hazard index, erosion hazard class, and erosion hazard level could be used as an indicator to determine the erosion status in the area. The hydro-orological parameters recorded in this study are shown in Table 7. The tolerable erosion rate for sloping lands is 25 ton ha $^{-1}$ year $^{-1}$ at a soil depth of more than 100 $\mathrm{cm}$ (Rahim 1995). The erosion rate of both steep slope and very steep slope plots were less than 15 ton ha ${ }^{-1}$ year $^{-1}$ while the soil depth in the plot was more than $90 \mathrm{~cm}$. The potential erosion rates in steep slope and very steep slope in studied plots were 0.38 ton $\mathrm{ha}^{-1}$ year $^{-1}$ and 1.31 ton $^{-1} \mathrm{hear}^{-}$ ${ }^{1}$, respectively. The erosion hazard index was 0.03 (low) and 0.13 (low) for steep slope and very steep slope plots, respectively. The erosion hazard classes (class I) and erosion hazard level in both studied slopes belong to "very low" category.

The soil erosion rates recorded in the current study was lower than the values reported for some other agroforestry 
systems (Karyati et al. 2018; Sarminah et al. 2018; Sumarno et al. 2011) as presented in Table 8. This result implied that the combination of vegetative ( $F$. moluccana and $V$. cylindrica) and terrace system could be effectively implemented in different sloping lands.

\section{Economic aspects}

The implementation of vegetative ( $F$. moluccana- $V$. cylindrica) and terrace system in different slope classes involves cost expenditure to buy planting and other materials, depreciation on equipment and wages of labor. On the other hand, implementation of this method gives revenue and profit, especially from $V$. cylindrica yield. Table 9 shows economic analysis of $V$. cylindrica as intercrop in vegetative ( $F$. moluccana-V.cylindrica) and terrace combination system in the two different slope classes. The material cost involved was for buying $F$. moluccana seedlings, V. cylindrica seeds, NPK fertilizer, pesticides, plastic strings, gunny sacks, and fertilizers to increase soil fertility. The profit of $F$. moluccana- $V$. cylindrica plantation in steep slope was $\mathrm{Rp} 3,865,000.00$ $\mathrm{ha}^{-1} \mathrm{cs}^{-1}$ and in very steep slope was Rp 665,000.00 ha- $\mathrm{cs}^{-}$ ${ }^{1}$. This profit was from selling the yield from $V$. cylindrica.

The profit from $V$. cylindrica yield in steep slope was higher than $A$. hypogaea yield on agroforestry system of $F$. moluccana-A. hypogaea as reported by Karmini et al. (2017). The total cost, total revenue and profit related to $F$. moluccana and A. hypogaea agroforestry system was $\mathrm{Rp}$ 10,985,000 ha $\mathrm{hs}^{-1}$, Rp 14,000,000 ha $\mathrm{cs}^{-1}$ and Rp $3,015,000 \mathrm{ha}^{-1} \mathrm{cs}^{-1}$. However, growing $V$. cylindrica as intercrop in vegetative ( $F$. moluccana-V.cylindrica) and terrace combination system in different slope lands is highly beneficial when compared to growing G. $\max$ in $A$. cadamba-G. max agroforestry system. Karmini et al. (2017) reported that growing G. max in A. cadamba-G. max agroforestry system involved total cost of $\mathrm{Rp}$ $11,019,000.00 \mathrm{ha}^{-1} \mathrm{cs}^{-1}$, total revenue was $\mathrm{Rp} 3,500,000 \mathrm{ha}^{-}$ ${ }^{1} \mathrm{cs}^{-1}$, and the profit was Rp (-)7,519,000.00 $\mathrm{ha}^{-1} \mathrm{cs}^{-1}$. The planting arbor trees and shrubs densely on the terrace riser slope could suppress gravity erosion, allow intensive cultivation, add economic benefits and extend development of the landscape efficiently (Cao et al. 2007).

The implementation of a combination of vegetative and terrace system for soil and water conservation could be applied in the different slope lands. This practice will be beneficial based on silvicultural, hydro-orological, and economic aspects. The short term economic benefit is provided by agricultural yield from the crop plant. In long term, this practice is beneficial in terms of soil and water conservation and environment aspects. The information of this study on silvicultural, hydro-orological and economic aspects could be used for recommending alternative agroforestry systems in small scale, and soil conservation programs in a wide-scale for all stakeholders including farmers, foresters, private parties and the governmental agencies concerned with land management and soil conservation activities.

\section{ACKNOWLEDGEMENTS}

The authors wish to express their thanks to Ministry of Research, Technology and Higher Education, Republic of Indonesia for supporting Hibah Penelitian Strategis Nasional Institusi Contract No.: 140/UN17.41/KL/2018.

\section{REFERENCES}

ADB. 2016. Indonesia Country Water Assessment. Manila. (PATA 8432 INO). ADB, Mandaluyong City, Philippines.

Bocco G, Napoletano BM. 2017. The prospects of terrace agriculture as an adaptation to climate change in Latin America. Geography Compass. 2017;11:e12330. DOI: $10.1111 /$ gec 3.12330

Bray RH. 1954. A nutrient mobility concept of soil-plant relationships. Soil Sci 78 (1): 9-22.

Cao S, Chen L, Feng Q, Liu Z. 2007. Soft-riser bench terrace design for the hilly loess region of Shaanxi Province, China. Landsc Urban Plan 80: 184-191.

Chaplot V, LeBissonnais Y. 2000. Field measurements of interrill erosion under different slopes and plot sizes. Earth Surf Proces Landf 25: $145-153$.

Dass A, Sudhishri S, Lenka NK, Patnaik US. 2011. Runoff captured through vegetative barriers and planting methodologies to reduce erosion, and improve soil moisture, fertility and crop productivity in southern Orissa, India. Nutr Cycl Agroecosyst 89:45-57.

Fisher RF, Binkley D. 2000. Ecology and Management of Forest Soils. $3^{\text {rd }}$ ed. John Wiley \& Sons, Inc, USA.

Hammer WI. 1981. Second soil conservation consultant report. AGOF/INS/78/006. Tech. Note No. 10. Centre for Soil Research, Bogor.

Hamzah MZ, Arifin A, Zaidey AK, Azirim AN, Zahari I, Hazandy AH. 2009. Characterizing soil nutrient status and growth performance of planted dipterocarp and non-dipterocarp species on degraded forest land in Peninsular Malaysia. J Appl Sci 9 (24): 4215-4223.

Harris RW. 1992. Arboriculture: Integrated Management of Landscape Trees, Shrubs, and Vines. Prentice-Hall Career \& Technology, New Jersey.

Hazra P, Som MG. 2006. Vegetable Science. Kalyani Publishers, New Delhi.

Karmini, Sarminah S, Karyati. 2017. Economic analysis of groundnut (Arachis hypogaea) and soybean (Glycine max) as intercropping plants in two agroforestry systems. Biodiversitas 18 (2): 483-493.

Karyati, Ardianto S. 2016. Dinamika suhu tanah pada kedalaman berbeda di Hutan Pendidikan Fakultas Kehutanan Universitas Mulawarman. Jurnal Riset Kaltim 4 (1): 1-12. [Indonesian]

Karyati, Sarminah S, Karmini, Simangunsong G, Tamba J. 2018. The mixed cropping of Anthocephalus cadamba and Glycine max for rehabilitating sloping lands. Biodiversitas 19 (6): 2088-2095.

Kjeldahl J. 1883. New method for the determination of nitrogen. Chem News 48 (1240): 101-102.

Kumar JIN, Kumar RN, Bhoi RK, Sajish PR.2010. Tree species diversity and soil nutrient status in three sites of tropical dry deciduous forest of western India. Trop Ecol 51 (2): 273-279.

Ministry of Agriculture Republic of Indonesia. 2018. Agricultural Statistics 2018. Ministry of Agriculture Republic of Indonesia, Jakarta. [Indonesia]

Nizam MS, Norziana J, Sahibin AR, Latiff A. 2006. Edaphic relationships among tree species in the National Park at Merapoh, Pahang, Malaysia. J Bioscience 17 (2): 37-53.

Nooprom K, Santipracha Q. 2015. Effect of varieties on growth and yield of yard long bean under Songkhla Conditions, Southern Thailand. Modern Appl Sci 9 (13): 247-251.

Nyssen J, Poesen J, Deckers J. 2008. Land degradation and soil and water conservation in tropical highlands. Soil Tillage Res. DOI:10.1016/j.still.2008.08.002

Potts MD, Ashton PS, Kaufman LS, Plotkin JB. 2002. Habitat patterns in tropical rain forest: A comparison of 105 plots in northwest Borneo. Ecology 83 (10): 2782-2797. 
Rahim SE. 1995. Erosi Tanah dan Pemodelan Pendugaannya dalam Dinamika Lingkungan Hidup Sumatera Selatan. PPLH Lembaga Penelitian Universitas Sriwijaya, Palembang. [Indonesian]

Regulation of Ministry of Forestry Republic of Indonesia, Ministry of Forestry Republic of Indonesia Number: P.60/Menhut-II/2009. [Indonesian]

Regulation of Directorate General of Watershed Management and Social Forestry, Ministry of Forestry Republic of Indonesia Number: P.4/VSET/2013. [Indonesian]

Sarminah S, Karyati, Karmini, Simbolon J, Tambunan E. 2018 Rehabilitation and soil conservation of degraded land using sengon (Falcataria moluccana) and peanut (Arachis hypogaea) agroforestry system. Biodiversitas 19 (1): 222-228.

Schmidt FH, Ferguson JHA. 1951. Rainfall types based on wet and dry period ratios for Indonesia and western New Guinea. Kementerian Perhubungan Djawatan Meteorologi dan Geofisik, Verhandelingen No. 42. Jakarta.

Slavin SL. 2009. Economics. McGraw-Hill Irwin, New York.

Sumarno, Winarno J, Prastomo I. 2011. Kajian pengelolaan lahan berdasarkan tingkat bahaya erosi dan pola konservasi tanah dan air di Desa Ngadipiro Kecamatan Nguntoronadi, Kabupaten Wonogiri. Sains Tanah-Jurnal Ilmu Tanah dan Agroklimatologi 8 (1): 13-22. [Indonesian]
Swestiani D, Purwaningsih S. 2013. Produksi kacang tanah (Arachis hypogaea L.) pada agroforestri berbasis kayu sengon dan manglid. Jurnal Penelitian Agroforestri 1 (2): 71-82. [Indonesian]

Statistics of East Kalimantan Province. 2018. Kalimantan Timur Province in Figure 2018. BPS Statistics of East Kalimantan Province, Samarinda. [Indonesian]

Tanaka S. 1999. Soil ecological study on shifting cultivation in the mountains area in Northern Thailand. Faculty of Agriculture, Kochi University. Nankoku, Japan.

Toledo M, Poorter L, Peña-Claros M, Alarcón A, Balcázar J, Leaño C, Licona JC, Llanque O, Vroomans V, Zuidema P, Bongers F. 2011. Climate is a stronger driver of tree and forest growth rates than soil and disturbance. J Ecol 99: 254-264.

Wahyu I, Pranoto H, Supriyanto B. 2018. Kajian produktivitas tanaman semusim pada sistem agroforestri di Kecamatan Samboja Kabupaten Kutai Kartanegara. Agroekoteknologi Tropika Lembab 1 (1): 24-33. [Indonesian]

Wahyudi, Panjaitan S. 2013. Perbandingan sistem agroforestri, monokultur intensif, dan monokultur konvensional dalam pembangunan hutan tanaman sengon. Prosiding Seminar Nasional Agroforestri 165-171. [Indonesian]

Walkley A, Black IA. 1934. An examination of the Degtjareff method for determining soil organic matter and a proposed modification of the chromic acid titration method. Soil Sci 37: 29-38. 